\title{
Operational Status of the Superconducting System for LHD
}

\author{
Toshiyuki Mito, Arata Nishimura, Shuichi Yamada, Shinsaku Imagawa, Kazuya Takahata, Nagato Yanagi, \\ Hirotaka Chikaraishi, Hitoshi Tamura, Ryuji Maekawa, and Osamu Motojima
}

\begin{abstract}
Large Helical Device (LHD) is a heliotron-type experimental fusion device which has the capability of confining current-less and steady-state plasma. The primary feature on the engineering aspect of LHD is using superconducting (SC) coils for magnetic confinement: two pool boiling helical coils (H1, H2) and three pairs of forced-flow poloidal coils (IV, IS, OV). These coils are connected to the power supplies by SC bus-lines. Five plasma experimental campaigns have been performed successfully in four years from 1998. The fifth operation cycle started in August 2001 and finished in March 2002. We have succeeded to obtain high plasma parameters such as $10 \mathrm{keV}$ of electron temperature, $5 \mathrm{keV}$ of ion temperature and beta value of $3.2 \%$. The operational histories of the SC coils, the SC bus-lines and the cryogenic system have been demonstrating high reliability of the large scale SC system. The operational status and the results of device engineering experiments are summarized.
\end{abstract}

Index Terms-Fusion, large helical device (LHD), large scale, superconducting coils.

\section{INTRODUCTION}

$\mathbf{N}$ ATIONAL Institute for Fusion Science (NIFS) was established in 1989 as an inter-university research institute in Japan, to study the science of nuclear fusion and its application for the realization of a future nuclear fusion reactor. The Large Helical Device (LHD) project has been initiated at NIFS. The LHD is an experimental fusion device which uses the heliotron magnetic field concept developed in Japan. In order to confine current-less steady-state plasma, LHD was designed as a fully superconducting (SC) system consisting of two helical coils, three pairs of poloidal coils and SC bus-lines. The construction of LHD started in 1991 by manufacturing the helical coil winding machine, the IV poloidal coil, the LHD main experimental building. The LHD construction was completed by the end of 1997 as an eight-year project. The plasma experiment was started on March 31, 1998, just as originally scheduled at the beginning [1]. Since then, five experimental campaign cycles have been performed in four years and we have already succeeded in obtaining high plasma parameters which substantially exceed those of the previous helical type devices.

\section{FEATURES OF LHD}

\section{A. Overview of $L H D$}

Fig. 1 shows the LHD cryostat surrounded by plasma heating systems (NBI, ECH, ICRF), plasma diagnostic systems,

Manuscript received August 6, 2002.

The authors are with the National Institute for Fusion Science (NIFS), Gifu-ken 509-5292, Japan (e-mail: mito@nifs.ac.jp).

Digital Object Identifier 10.1109/TASC.2003.812741

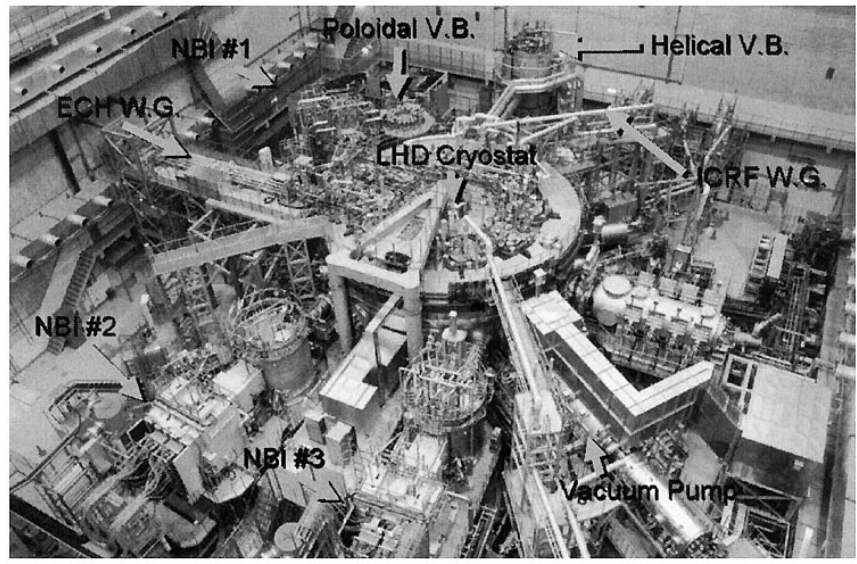

Fig. 1. Photograph of the Large Helical Device (LHD).

TABLE I

MAJOR SPECIFICATIONS OF LHD

\begin{tabular}{ll}
\hline \hline Plasma major radius & $3.5-4.1 \mathrm{~m}$ \\
Average plasma minor radius & $0.5-0.65 \mathrm{~m}$ \\
Plasma volume & $20-30 \mathrm{~m}^{3}$ \\
Toroidal magnetic field & $3 \mathrm{~T}$ \\
Field period & 10 \\
Coil stored energy & $0.9 \mathrm{GJ}$ \\
Cryostat & $\phi 13.5 \mathrm{~m} \times 8.8 \mathrm{~m}$ \\
Cold mass & $820 \mathrm{t}$ \\
Total weight & $1500 \mathrm{t}$ \\
\hline
\end{tabular}

vacuum pumping system, and control-valve-boxes of the cryogenic system, etc. in the LHD experimental room. Major specifications of LHD are listed in Table I [2], [3].

\section{B. Superconducting System for LHD}

A pair of helical coils $(\mathrm{H} 1, \mathrm{H} 2)$ with toroidal pitch number 10 has major and minor radii of $3.9 \mathrm{~m}$ and $0.975 \mathrm{~m}$, respectively. A helical coil consists of three blocks, the Aluminum stabilized $\mathrm{NbTi} / \mathrm{Cu}$ pool boiling type conductor wound from $\mathrm{H}-\mathrm{I}$ (inner) from 8 layers followed by $\mathrm{H}-\mathrm{M}$ (middle) and $\mathrm{H}-\mathrm{O}$ (outer) with 6 layers respectively. The windings were covered with thick 316 stainless steel case designated a HC cans to sustain large electromagnetic forces acting in the windings. The $\mathrm{HC}$ cans are also used as a vessel of liquid helium. Three pairs of forced-flow poloidal coils are named the Inner Vertical (IV) coils, the Inner Shaping (IS) coils and the Outer Vertical (OV) coils. Each pair of coils consists of an upper (U coil) and a lower coil (L coil). Each coil consists of eight double-pancakes wound with $\mathrm{NbTi} / \mathrm{Cu}$ cable-in-conduit conductors (CICC). Supercritical helium is supplied to each pancake in parallel from inner 


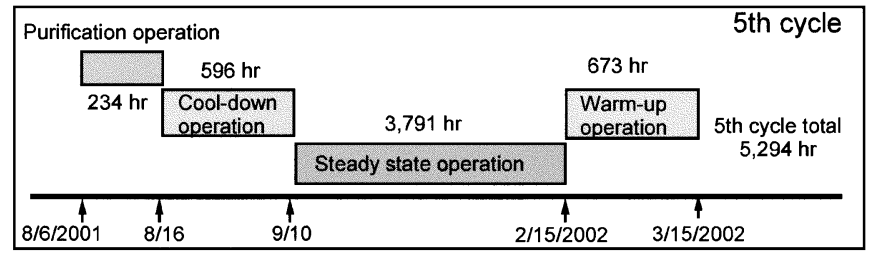

Fig. 2. Fifth cycle operation of LHD.

turns to the outer turns. The helical and poloidal coils are assembled into the supporting structure which sustains the large electromagnetic forces acting on the coils.

Flexible SC bus-lines, approximately 49 to $62 \mathrm{~m}$ long, connect SC coils and power supplies. One helical coil is electrically separated into three blocks and there are six blocks in the helical coils (H1-I, H1-M, H1-O, H2-I, H2-M, H2-O). On the other hand, each pair of upper and lower poloidal coils (IV, IS and $\mathrm{OV}$ ) are connected in series inside the cryostat. The number of SC bus-lines is nine (six for the helical coils and three for the poloidal coils) and their total length is $497 \mathrm{~m}$ with a maximum operating current of $31.3 \mathrm{kA}$. It should be noted that this is one of the innovative components newly applied to a fusion device introduced by the LHD project [4].

\section{OPERATION OF LHD}

\section{A. Cryogenic System}

The helium refrigerator/liquefier has a cooling capacity of $5.67 \mathrm{~kW}$ at $4.4 \mathrm{~K}, 20.6 \mathrm{~kW}$ from $40 \mathrm{~K}$ to $80 \mathrm{~K}$ and $650 \mathrm{l} / \mathrm{h}$ liquefaction, simultaneously. Refrigerated objects are the helical coils with liquid helium pool boiling and the poloidal coils with supercritical helium forced flow cooling, the support structure and the SC bus-lines with two phase helium forced flow cooling and the self-cooling current leads with vaporized liquid helium. Three kinds of cryogen (supercritical helium, liquid helium, $40 \mathrm{~K}-80 \mathrm{~K}$ helium gas for radiation shields), which are supplied from the cold box, are distributed and controlled by the helical valve box, the poloidal valve box and the current leads cryostats as the junction boxes for the SC bus-lines. Full automatic control for the various cooling modes such as, a cool-down, a steady-state, a warm-up, a power failure, a coil quench, has been implemented into the cryogenic control system (LHD-TESS) [5]. Safety and stable operation of the LHD cryogenic system is crucial since LHD is not a test system for a large-scale SC application but an experimental device to study fusion plasma.

\section{B. Fifth Cycle Operation}

The fifth cycle operation of the LHD is summarized in Fig. 2. Operating cycle consists of purification, cool down, steady state, and warm-up operations. In the fifth operating cycle, the compressor started on August 6, 2001 and the system purification operation was performed for 10 days (234 hours). A criterion of $2 \mathrm{ppm}$ or less for the impurity concentration (such as oxygen, nitrogen, water, etc.) had been set for the completion of the purification process criteria. The cool down started on August 16 and finished on September 9 for 25 days (596 hours) as shown in Fig. 3. The steady-state operation was continued from

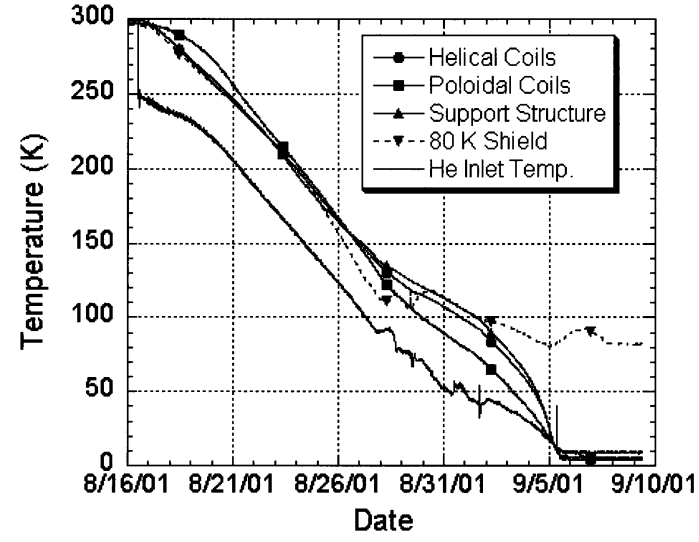

Fig. 3. Cool down curves of the LHD fifth cycle operation.

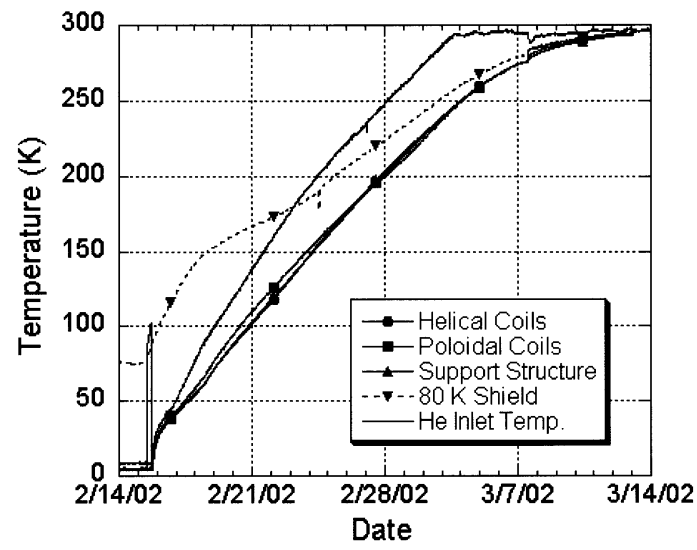

Fig. 4. Warm up curves of the LHD fifth cycle operation.

September 10 to February 14, 2002 for 158 days (3791 hours). The warm-up operation for the entire system started on February 15, and ended on March 15, 2002 for 28 days (673 hours) as shown in Fig. 4. Total operation time of the fifth cycle was 220 days (5294 hours).

\section{Operating History of the Previous Cycles}

The integrated operation time for four years from 1998 has become 23745 hours by the end of fifth cycle operation, and it had realized the high operating ratio of two thirds of the total annual time as show in Fig. 3.

High reliability for long-term operations is required because a plasma experimental campaign will continue several months and a warm-up of the total SC system will be done only once per year. During the operations for four years, in order to upgrade the reliability of the LHD system, various improvements have been carried out in nonoperational periods. For example; 1) extension of helium buffer tanks for making the inventory of helium gas increase, 2) addition of a recovery compressor and extension of recovery lines for collecting the evaporated helium gas at the time of the main compressors emergency shut down by power failure etc., 3 ) addition of the fifth oil separator unit of the main compressors for reducing the amount of oil contamination to the cold box, 4) program corrections of the cryogenic control system and improvement of its hardware reliability.

The count of the malfunctions/abnormalities which resulted in stoppage of the cryogenic system is listed in Table II. The 

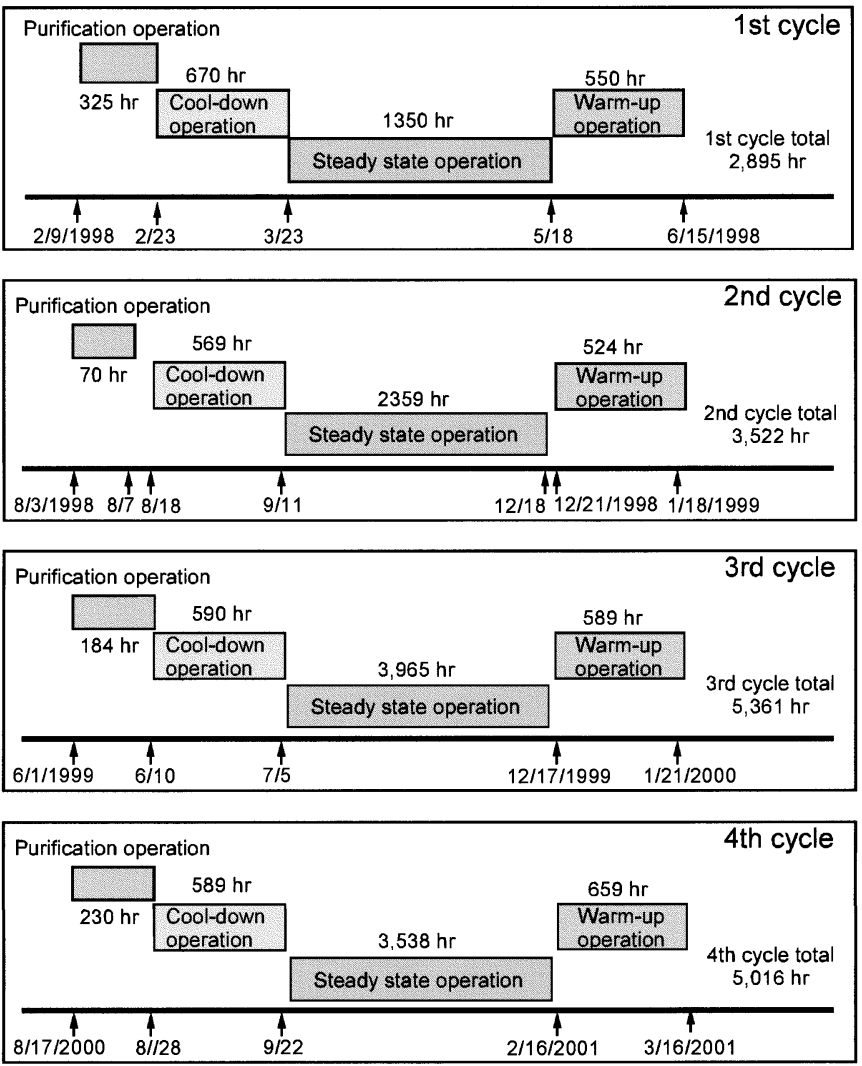

Fig. 5. Operating History of LHD for the Previous Cycles.

TABLE II

The Count of the MaLfunctions/ABNORMalities Which Resulted IN A STOPPAGE OF THE CRYOGENIC SYSTEM

\begin{tabular}{lcccc}
\hline \hline \multicolumn{1}{c}{ Operation } & Cool-down & Steady state & Warm-up & Sub-total \\
\hline First cycle & $0(0)$ & $2(0)$ & $1(0)$ & $3(0)$ \\
Second cycle & $2(2)$ & $1(0)$ & $0(0)$ & $3(2)$ \\
Third cycle & $0(0)$ & $3(2)$ & $0(0)$ & $3(2)$ \\
Forth cycle & $0(0)$ & $0(0)$ & $1(0)$ & $1(0)$ \\
Fifth cycle & $0(0)$ & $1(1)$ & $0(0)$ & $1(1)$ \\
\hline Total & $2(2)$ & $7(3)$ & $2(0)$ & $11(5)$ \\
\hline O) shows the count of the stoppage due to electric power failure
\end{tabular}

total count in which the cryogenic system made an abnormal stoppage during the operation cycles was 11 times in four years. Restoring the normal operation status in a short time has been made possible by an implementation of the automatic sequence program corresponding to emergency situations such as electric power failure and quick repair of a malfunction part. Furthermore, in the 4th and the 5th cycle, there were no stoppages during the steady-state, and stable operation has been continued. There have been no serious problems in the LHD SC system that caused a long stoppage of the plasma experiments. The high reliability of this large-scale SC system has been demonstrated.

The stoppages due to electric power failure happened 5 times among the 11 stoppages of the cryogenic system. Most of these electric power failures were less than 1 second. Aiming at the further stable operation, the installation of SMES has been considered as an Uninterruptible Power Supply (UPS) which can compensate 3.5 MW for 2 seconds.
TABLE III

MAXIMUM ACHIEVEd Plasma PARAMETERS

\begin{tabular}{lrl}
\hline \multicolumn{1}{c}{ Item } & Achievements \\
\hline Line-average density & $1.5 \times 10^{20}$ & $\mathrm{~m}^{-3}$ \\
Energy confinement time & 0.36 & $\mathrm{~s}$ \\
Plasma stored energy & $1.16 \mathrm{MJ}$ \\
Electron temperature & $10 \mathrm{keV}$ \\
Ion temperature & $5.0 \mathrm{keV}$ \\
Volume-average beta & $3.2 \%$ \\
Discharge duration & $127 \mathrm{~s}$ \\
\hline
\end{tabular}

TABLE IV

CURRENTS OF COILS For FundAMENTAL EXCITATION MODES AT 3 T

\begin{tabular}{ccccc}
\hline mode & HC $[\mathrm{kA}]$ & OV $[\mathrm{kA}]$ & IS $[\mathrm{kA}]$ & IV [kA] \\
\hline$\# 1-\mathrm{o}$ & 12.5 & -19.62 & -5.01 & 10.21 \\
$\# 1-\mathrm{a}$ & 12.5 & -21.74 & 5.24 & 5.74 \\
$\# 1-\mathrm{b}$ & 12.5 & -17.49 & -15.27 & 14.68 \\
$\# 1-\mathrm{c}$ & 13.0 & -19.60 & -6.80 & 8.09 \\
$\# 1-\mathrm{d}$ & 12.0 & -19.60 & -3.28 & 12.25 \\
\hline
\end{tabular}

\section{ACHIEVEMENTS OF THE Plasma ExPERIMENTS}

In order to reach the goal of the highly efficient plasma confinement, the heating powers have been increased up to $9 \mathrm{MW}$ for NBI, 1.9 MW by ECH and 2.7 MW by ICH. We have succeeded in obtaining the high plasma parameters such as $10 \mathrm{keV}$ of electron temperature, $5 \mathrm{keV}$ of ion temperature, $1.16 \mathrm{MJ}$ of stored energy and beta value of $3.2 \%$ as listed in Table III.

\section{DeVICE ENGINEERING EXPERIMENTS OF LHD}

\section{A. SC Coil Excitation Circuit}

The coil excitation circuit consists of six DC power supplies, nine sets of current-leads and superconducting bus-lines. The power supply contains thyristor rectifiers, DC filter, quick dump circuit, quench protection circuit and polarity change unit [6]. For quench detection, balance voltages are measured between each block (or each coil) connected in series. For example, the $\mathrm{H}-\mathrm{I}$ balance voltage is given by subtracting the terminal voltage of the H2-I block from that of the H1-I block in a quench detection circuit.

There are five fundamental operating modes for LHD as listed in Table IV. The mode named \#1-o, a, b, c and d, which correspond to the shapes of magnetic surfaces of quasicircle, vertical elongation, horizontal elongation, outward shift and inward shift, respectively. The major radius of the plasma axis is $3.75 \mathrm{~m}$ for \#1-o, a, b, 3.75-4.1 m for \#1-c, and 3.5-3.75 m for \#1-d.

\section{B. Excitation Tests Before the Plasma Experiments}

The excitation tests before the fifth cycle plasma experiment were conducted from September 11 to September 18, 2001. The following test results were attained.

〈 3 blocks of helical coils are at the same current

- \#1-o, $B=2.72 \mathrm{~T} @ 3.75 \mathrm{~m}$ (H-I/M/O:11.33 kA)

- \#1-d, $B=2.825$ T @ 3.60 m (H-I/M/O:11.30 kA)

$\langle 3$ blocks of helical coils are at different currents $\rangle>$

- \#1-d, $B=2.91 \mathrm{~T} @ 3.60 \mathrm{~m}$

$(\mathrm{H}-\mathrm{I} / \mathrm{M} / \mathrm{O}=10.975 / 11.873 / 12.072 \mathrm{kA})$

- \#1-d, $B=2.993$ T @ $3.50 \mathrm{~m}$

$(\mathrm{H}-\mathrm{I} / \mathrm{M} / \mathrm{O}=10.975 / 11.873 / 12.072 \mathrm{kA})$. 
The higher magnetic field can be achieved by grading the current of each block of the helical coils with the change of the helical pitch number $\gamma$. By succeeding in raising the magnetic field to $2.993 \mathrm{~T}$ at the plasma axis of $3.50 \mathrm{~m}$, plasma center heating at $3.5 \mathrm{~m}$ using electron cyclotron resonance heating $(\mathrm{ECH})$ was attained, and plasma with the high electron temperature of $10 \mathrm{keV}$ has been achieved successfully.

Other magnetic configurations were the same values as the highest value obtained in the previous cycles. It has been checked that there was no abnormal behavior, such as degradation in performance of the LHD SC system [7].

\section{Device Engineering Experiments}

The device engineering experiments were conducted during the fifth operation cycle for the purpose of determining the precise characteristics of the LHD SC system. The following new parameters and characteristics were obtained.

Helical Coils:

- Achieved a field of $2.917 \mathrm{~T} @ 3.60 \mathrm{~m}(\gamma=1.258)$, \#1-d without a local normal transition and recovery of the helical coils.

- In all excitation tests during the fifth cycle operation, there were no observations of a local normal transition and recovery in the balance voltages of the helical coils.

- Pulse height measurement of balance voltage showed that it had the tendency for a mechanical disturbance in the helical coils to decrease by repeating an operation cycle for a "training" effect [8].

- Spike signals accompanying $\gamma$ change were observed in the helical balance voltage. Since it is reproducible, there is the possibility of a magnetic instability (flux jump), which is a future investigation.

Poloidal Coils:

- Precise measurement of AC loss has been carried out and the following characters of the coupling loss became clear.

- The difference of the coupling loss characteristic between upper and lower coils was observed even if the coils have the same configuration [9].

- Superposition of the coupling loss components with very long time constants were observed for all poloidal coils with the CICC.

Power Supplies: By application of the H-infinity controller as a control scheme to guarantee robustness for stability, the coil current control of a high speed and high gain has been attained.

\section{Cryogenic Support Structure:}

- Precise strain measurements of the supporting structures have been continually monitored and no significant change has been observed [10].

\section{SUMMARY}

LHD has been used for extensive plasma experimentation since 1998 with 8 months operational period in each year. Five experimental campaign cycles have been performed in four years. The high reliability of the large-scale SC system of the LHD has been demonstrated. In particular, it is was important that the successful excitation of the magnetic field at the plasma axis of $3.50 \mathrm{~m}$ to $2.993 \mathrm{~T}$ leads to the high electron temperature of $10 \mathrm{keV}$ generated by ECH in the fifth operation cycle. In all excitation tests during the fifth operation cycle, there were no observations of a local normal transition in the helical coils. There were no abnormalities, such as degradation of a performance, in the LHD SC system. Furthermore, it seems that the mechanical disturbances in the helical coils have been decreasing by repeated operating cycles as a "training" effect.

\section{ACKNOWLEDGMENT}

The authors would like to thank the many collaborators at the universities, laboratories, and manufacturers for their collaboration in the design, development, construction, and operation of the LHD. They would also like to thank the LHD group for their continue support.

\section{REFERENCES}

[1] A. Iiyoshi, S. Imagawa, and LHD group, "Design, construction and the first plasma experiments in the Large Helical Device," Fusion Engineering and Design, vol. 46, pp. 323-335, 1999.

[2] O. Motojima, N. Yanagi, and A. Nishimura, "Review of SC magnet technologies developed in LHD project," Journal of Nuclear Materials, 258-263, pp. 234-240, 1998.

[3] T. Mito et al., "Design, development and operation of superconducting system for LHD," in Proc. of 19th IEEE/NPSS Symposium on Fusion Engineering (SOFE), Atlantic City, NJ, USA, January 22-25, 2002, to be published.

[4] S. Yamada et al., "Overall operating characteristics of superconducting current-feeder system for the LHD," Advances in Cryogenic Engineering, vol. 45, pp. 1525-1532, 2000.

[5] R. Maekara et al., "LHD cryogenic-control system performance under various operating conditions," Advances in Cryogenic Engineering, vol. 45 , pp. 1339-1346, 2000.

[6] H. Chikaraishi et al., "Dc power system for mutual coupled large scale superconducting coils," Cryogenics, vol. 42, pp. 89-95, 2002.

[7] S. Imagawa et al., "Consideration for the conductor motions in the helical coils of the Large Helical Device," IEEE Trans. Appl. Superconduct., vol. 13, pp. XXXX-XXXX, June 2003.

[8] N. Yanagi et al., "Pulse height analysis of the spike signals on the balance voltage observed in the LHD helical coils," in Proc ICEC 18 (Mumbai 2000), 2000, pp. 179-182.

[9] K. Takahata et al., "Coupling losses in cable-in-conduit conductors for LHD poloidal coils," Fusion Engineering Design, to be published.

[10] A. Nishimura et al., "Soundness evaluation of cryogenic support structure of Large Helical Device," in 22nd SOFT, Helsinki, Finland, 2002, F 25. 